\title{
Air Valve Clearance Fault Diagnosis of Diesel Engine Based on Acoustic Signal Data Processing
}

\author{
Cao Shuhua \\ Transportation Equipments and Ocean Engineering \\ College \\ Dalian Maritime University \\ Dalian, China \\ e-mail: caoshcindy@163.com
}

\author{
Xu JiuJun \\ Transportation Equipments and Ocean Engineering \\ College \\ Dalian Maritime University \\ Dalian, China \\ e-mail: daliankangbao@126.com
}

\author{
Ning Dayong \\ Transportation Equipments and Ocean Engineering \\ College \\ Dalian Maritime University \\ Dalian, China \\ e-mail: ningdayong@dlmu.edu.cn
}

\begin{abstract}
Acoustic fault diagnosis system of diesel engine is based on the analysis of the measured sound signals and try to extract the fault information contained in the signals which can be able to characterize the fault, and the contents of the research relates to the separation and feature extraction of the sound signal. In this paper, the research object is the diesel engine air valve abnormal sound signal. In order to identify the fault type, some digtal signal analysis methods such as digital filtering, wavelet transform and power spectral analysis method are adopted to extract and separate the abnormal sound signal. The results show that the methods owning some good analysis effects expressed as small amount of calculation and easy-to-real-time processing, which will contribute to the development of online sound fault detection device.
\end{abstract}

Keywords- fault diagnosis; acoustic signal data processing; diesel engine; digital filter; wavelet transform; spectral analysis

\section{INTRODUCTION}

Some parts of the diesel engine work in the harsh conditions as high temperature, high pressure and possess a higher probability of failure. In particular, the failure rate of the air valve is relatively high up to $11.9 \%$ [1]. The diesel engine sound contains information on the running state. Experienced staff can identify abnormal sound from the noisy sound and therefrom determine the type of failure. The key identifing the fault from abnormal sound lies in the operator familiar with these sound, but even for experienced operators, when the abnormal sound is weak or background noise is too loud, the discrimination of the fault will not be easy.
In this study, a real-time on-line diesel engine fault diagnosis system based on acoustic signal analysis is designed. The feasibility of the method is verified in the basis of the air valve abnormal sound signal. Its main task is to extract the frequency characteristics of the sound signal of air valve failure. When the diesel engine is running, a variety of acoustic signals mix together.Because these various failures is provided with different characteristic frequencies, it is possible to extract the characteristic frequency of sound signal by time-frequency analysis. Existing separation and extraction methods aim at sound characteristics involve cepstrum analysis, short-time Fourier transform, wavelet transform, and so on. And these methods have a successful application [2].

\section{THE IMPLEMENTATION OF THE SOUND SIGNAL DETECTION SYSTEM}

In this study, the fault that air valve clearance is too large is taken as the research object. The sound signals are collected during normal and abnormal operation of diesel engine. Appling the digital filtering, windowing function, wavelet transform and power spectral analysis, the collected sound signals are processed, and finally extracted the characteristic frequency of the abnormal noise signal.

\section{SIMULATION EXPERIMENTS AND DATA ANALYSIS}

\section{A The Fault sound signal acquisition}

In this paper, based on the composition of the signal acquisition system, a test stand has been built which can achieve real-time acquisition of the sound signal and data 
processing, using a microphone as a sound collection device, the sampling frequency being $44.1 \mathrm{kHz}$. The sound is collected as normal sound signal in the first experimental procedure when the the diesel engine is in the normal operation. And then the exhausting air valve clearance of the first cylinder is increased $0.4 \mathrm{~mm}$ and collected the sound as fault signal.

The normal sound signal is shown in Fig .1, and the abnormal sound signal is shown in Fig .2.

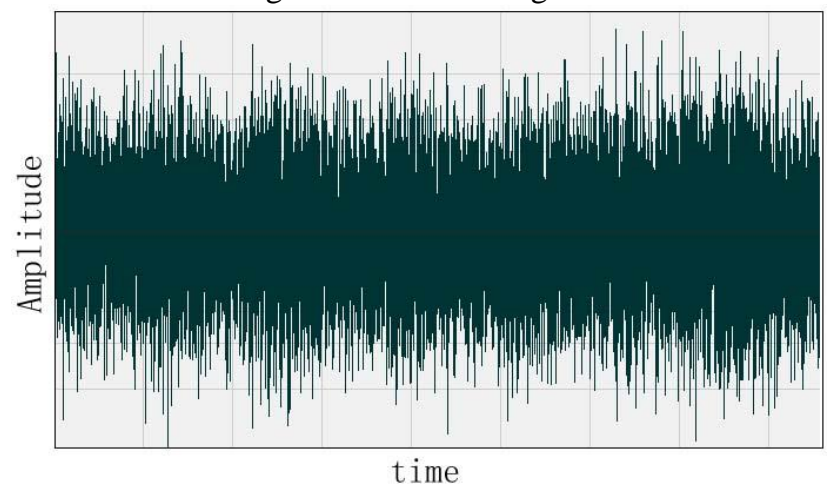

Figure 1. Normal sound signal

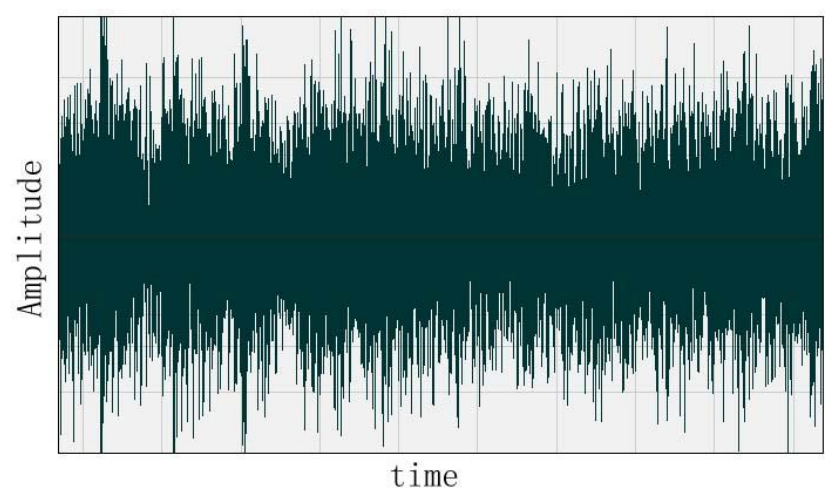

Figure 2. Fault sound signal

\section{B The data processing}

In the collected sound signal, it may be mixed with the ambient noise or other signal which must be removed. During the experiment, multiple sets of signal datas are measured in order to improve the accuracy and pertinence of the study. And the filter was applied to suppress noise [3 7].

\section{1) Signal filtering}

Signal filtering is one of the primary means of suppression and elimination of noise. And the signal can be frequency-selective through filter. So that the specific frequency component of the signal can be passed, while the other frequency component can be greatly attenuated. In the present experiment, the same filtering operation was performed on the normal sound signal and the failure sound signal. By manually adjusting the filter bandwidth repeatedly, the maximum different frequency band was found between the normal signal and the fault signal, which was identified as the characteristic frequency band of the fault sound.
The time domain curve of signal filtered is shown in Fig .3 and Fig.4.

\section{2) Appling window function}

The math tools commonly used in digital signal processing is the Fourier transform, which only is applicable to the analysis for the entire time domain and frequency domain. However, regarding to the computer signal processing, it is impossible to infinitely long signal processing, while analysis can only be taken a limited time segments. In order to short-term analysis, window function is applied to the signal processing [8].

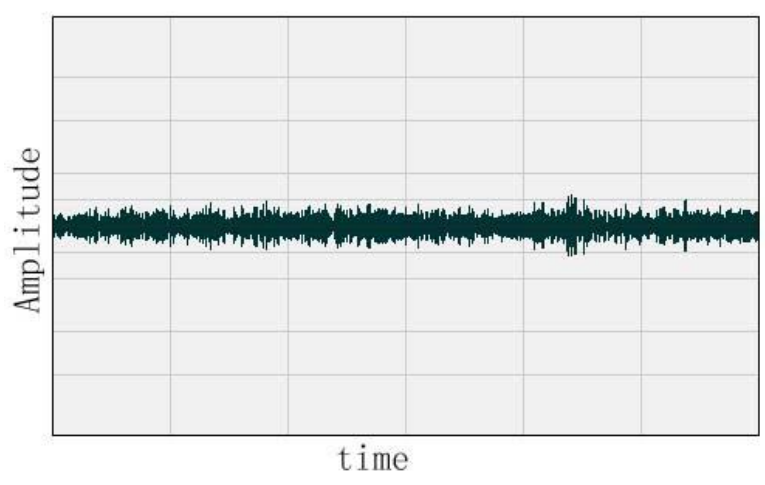

Figure 3. Normal sound signal

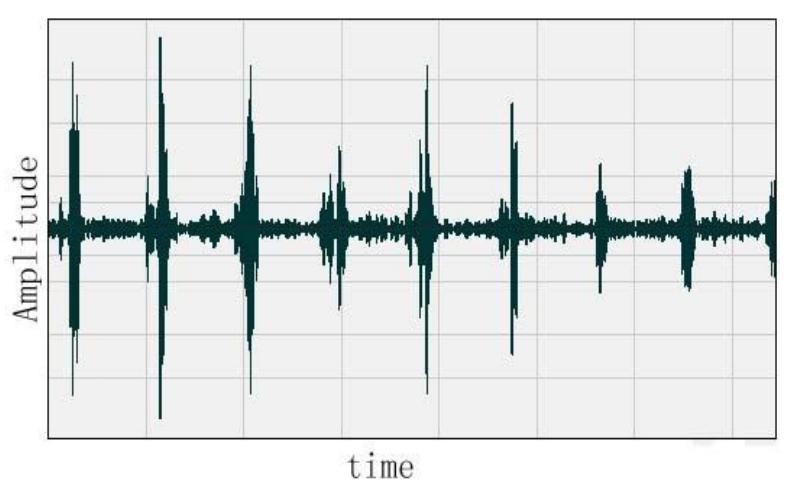

Figure 4. Fault sound signal

Once the signal is applied to the window function as well as the energy leakage will be produced.Therefore, when the window function is selected, we should consider the nature of the signal and processing requirements. The window function caused the energy leakage should have as little as possible, while not affect the frequency resolution. Taking into account the Hanning window is more suitable for the analysis of non-periodic continuous signal; the selected window function in this paper is the Hanning window.

Time-domain form of the Hanning window function can be expressed as

$$
w(k)=0.5\left(1-\cos \left(2 \pi \frac{k}{n+1}\right)\right) \quad k=1,2, \mathrm{~L}, n
$$

The frequency domain characteristics expressed as

$$
W(\omega)=\left\{0.5 W_{R}(\omega)+0.25\left[W_{R}\left(\omega-\frac{2 \pi}{N-1}\right)+W_{R}\left(\omega+\frac{2 \pi}{N-1}\right)\right]\right\}^{-\mathrm{j} \omega\left(\frac{N-1}{2}\right)}
$$


Where $W_{R}(\omega)$ is the amplitude frequency characteristic function of the rectangular window:

$$
W_{R}(\omega)=\frac{2 \sin \omega T}{\omega T}
$$

Where $\omega$ is angular frequency, $\mathrm{T}$ is the length of the window.

\section{3) Wavelet transform}

The wavelet transform is an analysis method in the time domain at the same time in the frequency domain, which has a good localization characteristic in the timefrequency domain. According to the different frequency components of the signal, wavelet transform can automatically adjust the density of the sampling $[9,10]$.

In this paper, the filtered sound signal by time was splited to several sections, whose each length is 1 second. Three sections signal each of from the normal signal and fault signal has been studied. Different wavelet bases were selected to analyze the sound signal wavelet decomposition, through comparative to select the proper wavelet. The final choice is $\mathrm{db} 10$ orthogonal wavelet bases, and then wavelet decomposition of four layers is performed to the signal. The results are shown in Fig .5and Fig .6.

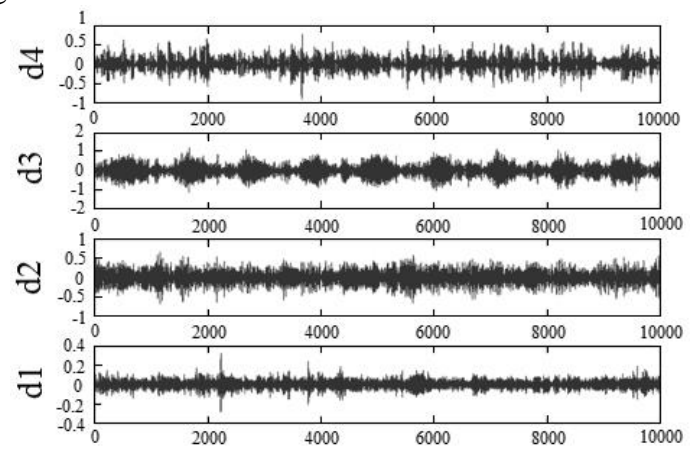

Figure 5. Db10 wavelet decomposition of the normal sound signal after filtering

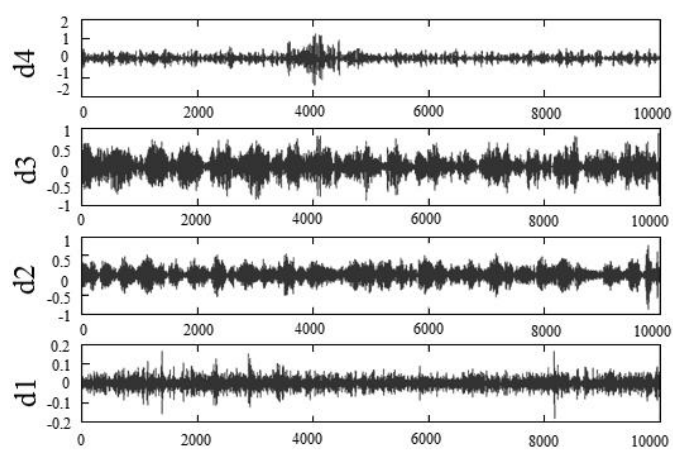

Figure 6. Db10 wavelet decomposition of the failure sound signal after filtering

\section{4) Power spectrum analysis}

By comparison of Fig .5 and Fig .6, it is difficult to distinguish between the normal signal and the fault signal. Thus, Power spectrum of the signal is obtained through further analytic signal, which can be expressed some statistical characteristics of the signal. In order to extract the characteristic frequency of the fault acoustic signal after filtering, the Hilbert envelope and the power spectrum analysis are executed for the detailed signal of the first layer d1 after the wavelet decomposition. As a result, shown by Fig .7 (a), (b), and (c).

Black as a normal signal power spectrum envelope line, gray as failure acoustic signal envelope as shown in Figure 7. Curve comparison of the two colors, it is easy to see that a clear distinction of power spectrum between the fault sound signals with normal sound signal. And three sections of the fault signal peak all appear at the location of $0.5 \times 104 \mathrm{~Hz}$, which fully explains the characteristic frequency of sound signal in trouble is about $5 \mathrm{KHz}$.
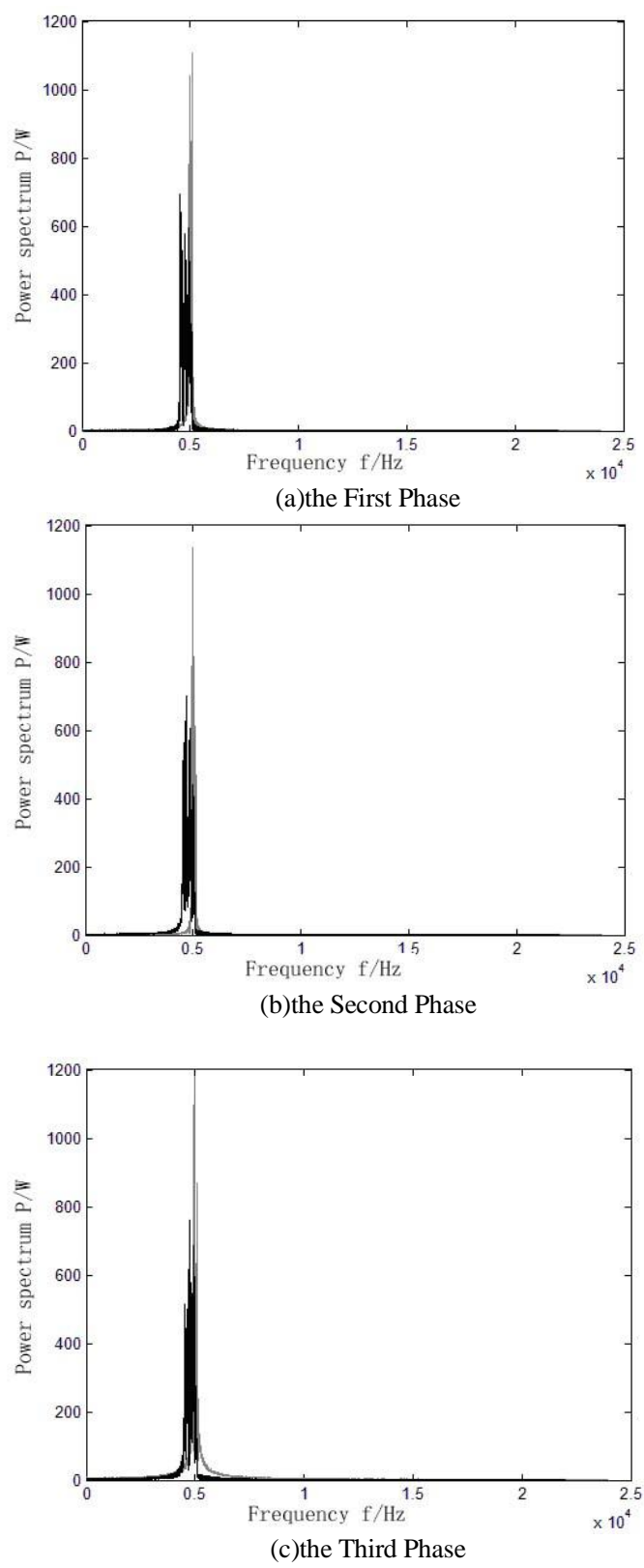

Figure 7. Power spectrum envelope

\section{CONCLUSION}

According to the above results, the following conclusions could be obtained:

Proposed diesel engine fault detection method based on the sound signal. The working status of the diesel engine 
can be judged based on the unusual sound. After the spectral analysis of the acoustic signal, the characteristic frequency of the signal is extracted as the basis to determine the fault.

Based on the Sound signal of "air valve clearance is too big", the collected sound signal is separated and extracted, and obtained the characteristic frequency is about $5 \mathrm{kHz}$.

In the paper, wavelet analysis and power spectrum analysis were integrated efficiently, since the power spectrum analysis can achieve the pure fault signal extraction as well as reduce the frequency components of the analyzed signal, filter off frequency interference of other signals. And wavelet analysis has the some advantages of acquiring mathematical details of signal. The experimental results show that the method is feasible and effective.

\section{REFERENCES}

[1] Chen Gang, Liao Mingfu. Fault Diagnosis for a Rolling Element Bearing Based on Wavelet Analysis. Science Technology and Engineering, 2007, vol. 7 no.12:2810-2814.

[2] Ghamry, M.El. Reuben, R. L. Steel, J. A. The development of automated pattern recognition and statistical feature isolation techniques for the diagnosis of reciprocating machinery faults using acoustic emission. Mechanical Systems and Signal processing, 2003,17:805-823.

[3] Yu Heji , Han Qingda, Li Shen. Equipments Fault Diagnosis Engineering. BeiJing. Metallurgical Industry Press, 2001.

[4] Zhou DongHua, Hu YanYan. Fault Diagnosis Techniques for Dynamic Systems. Acta Automatica Sinica, 2009, vol. 35 no. 6:748-758.

[5] Zhang Jin, Feng Zhipeng, Chu Fulei. Extraction of Rolling Bearing Fault Feature Based onTime-wavelet Energy Spectrum. Journal of Mechanical Engineering, 2011, vol. 47 no. 17:44-49.

[6] Wang Hongchao, Chen Jin, Huo Baiqi, et al. Noise-resistant Timefrequency Analysis Method and Its Application in Fault Diagnosis of Rolling Bearing. Journal of Mechanical Engineering, http://www.cnki.net/kcms/detail/11.2187.TH.20141016.0916.017.h tml.

[7] Xue Nianxi. Application of MATLAB in digital signal processing. BeiJing. Tsinghua University Press, 2008.

[8] Qin Ping, Fu Heping, Yan Bing. Diagnosing of contact friction fault of a plain bearing under dead load based on AE monitoring. Tribology, 2006, vol. 26 no.6:585-589.

[9] Jing Shuangxi, Tie Zhanxu,. Zhang Yingqi. Study for fault diagnosis techniques based on wavelet analysis. Journal of China Coal Society, 2000, 25:143-146.

[10] Liu Zengyong, Shi Baojie, Ma Hongwen, Analysized diesel air valve unusual inspection based on cylinder cap vibration signal Movable Power Station \& Vehicle, 2006,2:31-34. 